\title{
A DEGENERATIVE AFFLICTION
}

Multiple sclerosis often strikes between the ages of 20 and 40, when people are entering the workforce and raising families. By Charles Schmidt; illustration by Lucy Reading-Ikkanda.

\section{TURNING AGAINST THE SELF}

Multiple sclerosis is an incurable illness in which the body's own immune system destroys tissues in the central nervous system. T cells and B cells are thought to remove a protective coating called myelin that wraps around nerve fibres in the brain, spinal column and optic nerve. Exposed fibres are degraded, producing symptoms that vary depending on where the damage occurs.
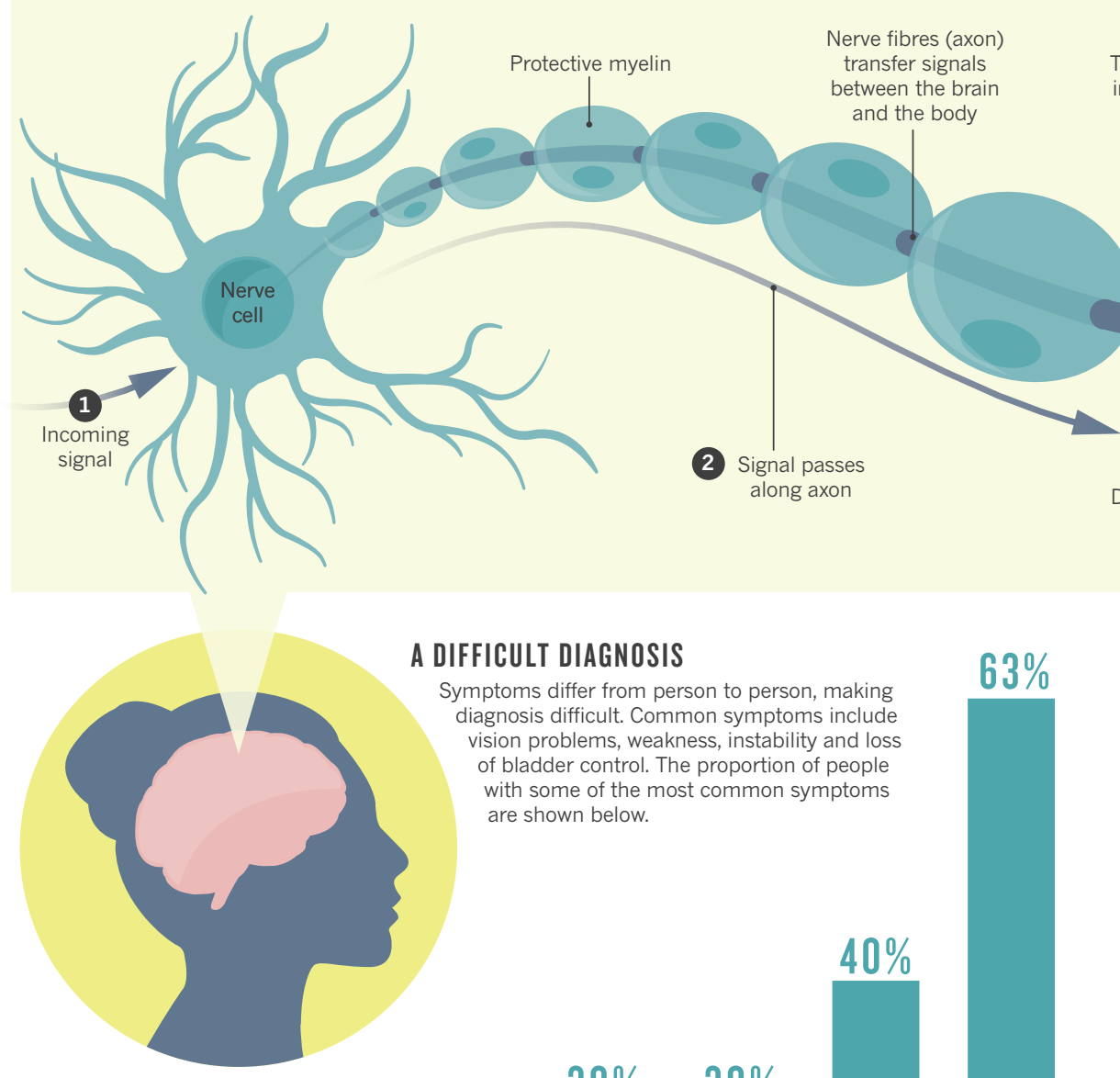

\section{A DIFFICULT DIAGNOSIS}

Symptoms differ from person to person, making diagnosis difficult. Common symptoms include vision problems, weakness, instability and loss of bladder control. The proportion of people with some of the most common symptoms are shown below.

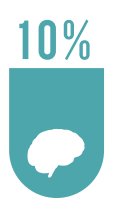

Cognitive Pain

Depression,

anxiety,

cognitive impairment, poor sleep and vertigo
Facial

pain,

burning,
Urinary

Urgency, nocturia, incontinence and hesitancy

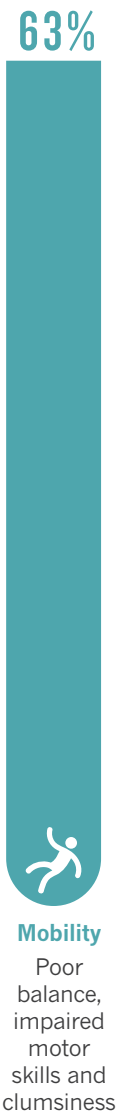

TYPES OF MULTIPLE SCLEROSIS

Relapsing-remitting multiple sclerosis (RRMS) produces attacks followed by periods of remission that can last months or years. It usually gets worse with time. In primary progressive multiple sclerosis (PPMS), symptoms worsen steadily without remission.

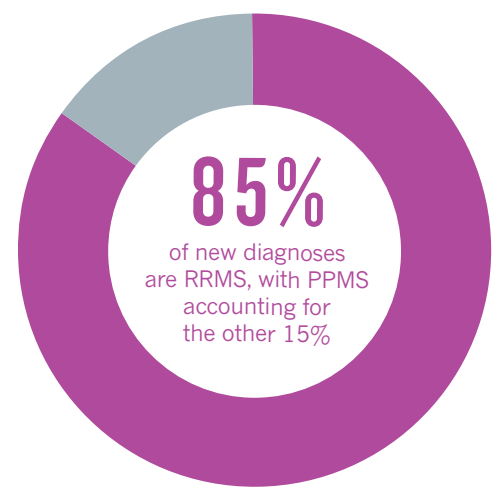

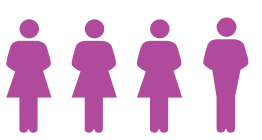

RRMS affects 2-3 TIMES as many women as men

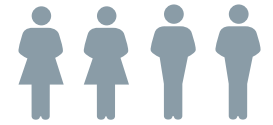

The number of women and men affected by PPMS is APPROXIMATELY EQUAL 


\section{WHAT CAUSES MULTIPLE SCLEROSIS?}

The cause is unknown, but it is thought to involve an interplay of genetics and the environment. Genetic susceptibility drives the risk, but the contribution of any specific gene seems to be modest.

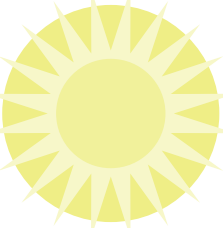

Environmental factors Living further from the Equator boosts risk, possibly because of reduced exposure to sunlight - the main source of vitamin $D$.

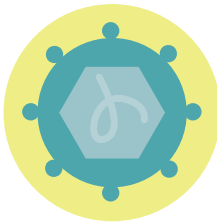

Epstein-Barr virus Few people who have been infected with the Epstein-Barr virus develop multiple sclerosis, but nearly everyone with the condition has been infected with the virus.

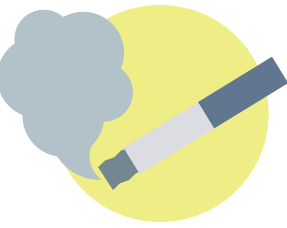

Smoking Numerous studies have found higher rates of multiple sclerosis in smokers than in non-smokers.

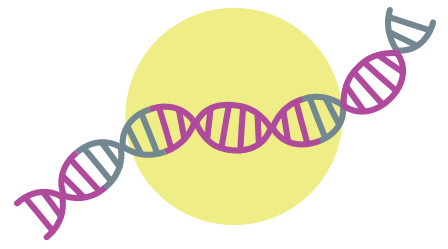

Genetic factors

Combined with vitamin $D$ deficiency, the HLA-DRB1*1501 allele and the CYP27B1 gene may elevate risk. IL2RA gene variants may increase the risk for individuals without a family history of the disease. IL7R variants are associated with PPMS.

\section{GLOBAL INCIDENCE}

Multiple sclerosis is most common in northern latitudes, reflecting the likely association with reduced exposure to sunlight and vitamin $\mathrm{D}$ deficiency.

Prevalance per country Number of people with multiple sclerosis per 100,000

$>100$ 5.01-20
$60.01-100$
$20.01-60$ No data

\subsection{MILLION}

Estimated number of people worldwide with the condition.
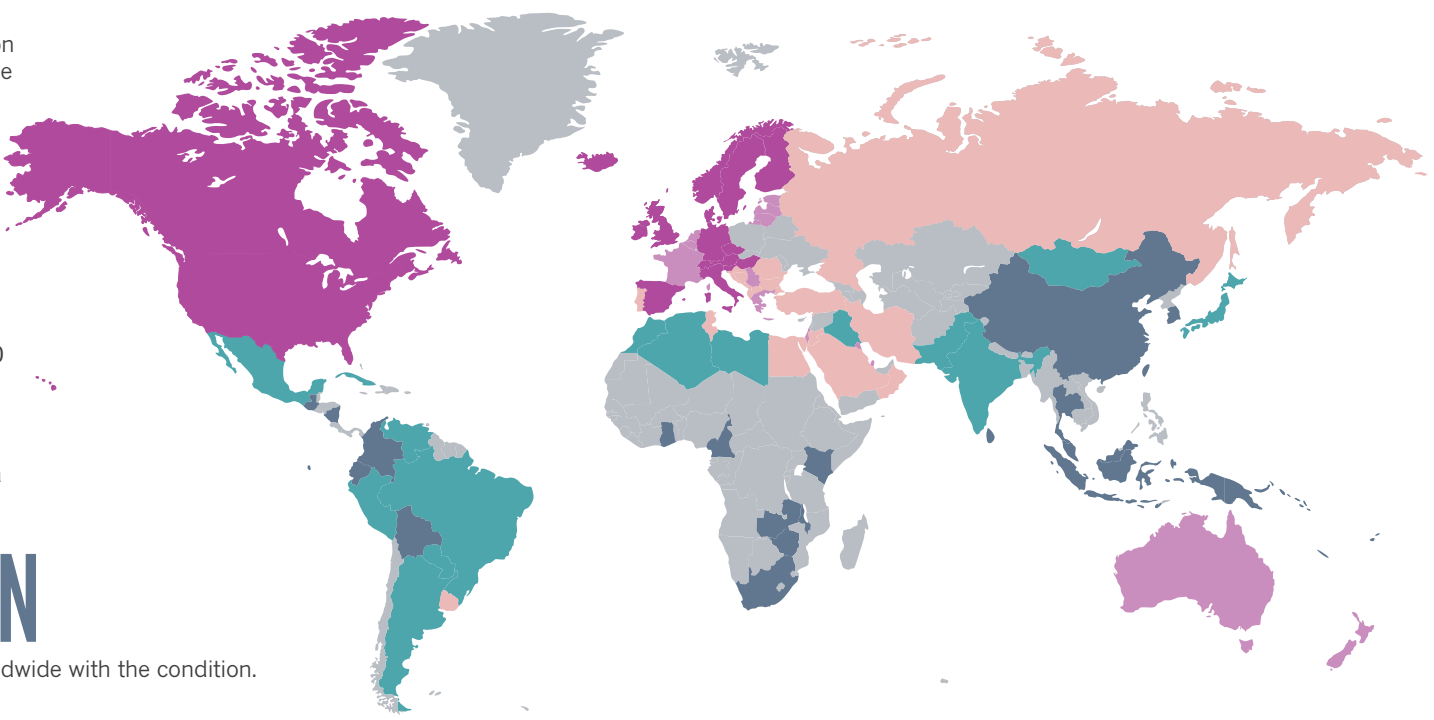

\section{DISEASE-MODIFYING THERAPIES}

Several drugs have been approved for RRMS, but none for PPMS. Drugs need to be given as soon as possible to slow the pace of neurological damage.

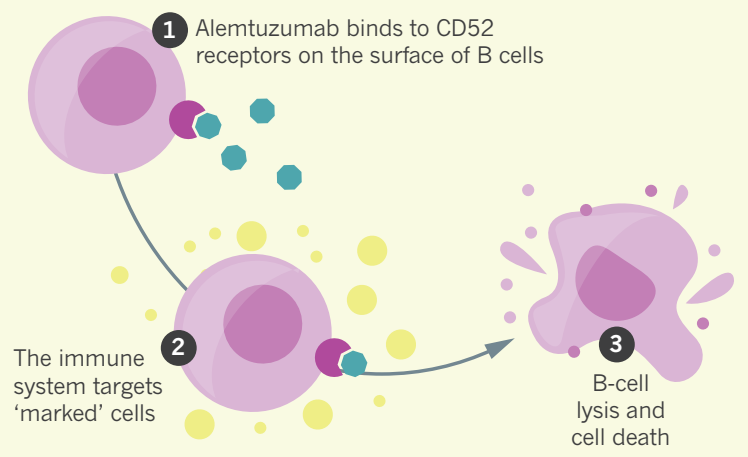

\section{ALEMTUZUMAB}

A monoclonal antibody also approved for leukaemia, alemtuzumab binds to CD52, a protein on the surface of mature B cells and T cells, which are then targeted for destruction by the immune system. Widespread B-cell depletion from alemtuzumab treatment may promote the growth of $T$ cells that are less autoreactive and do not cause inflammation.

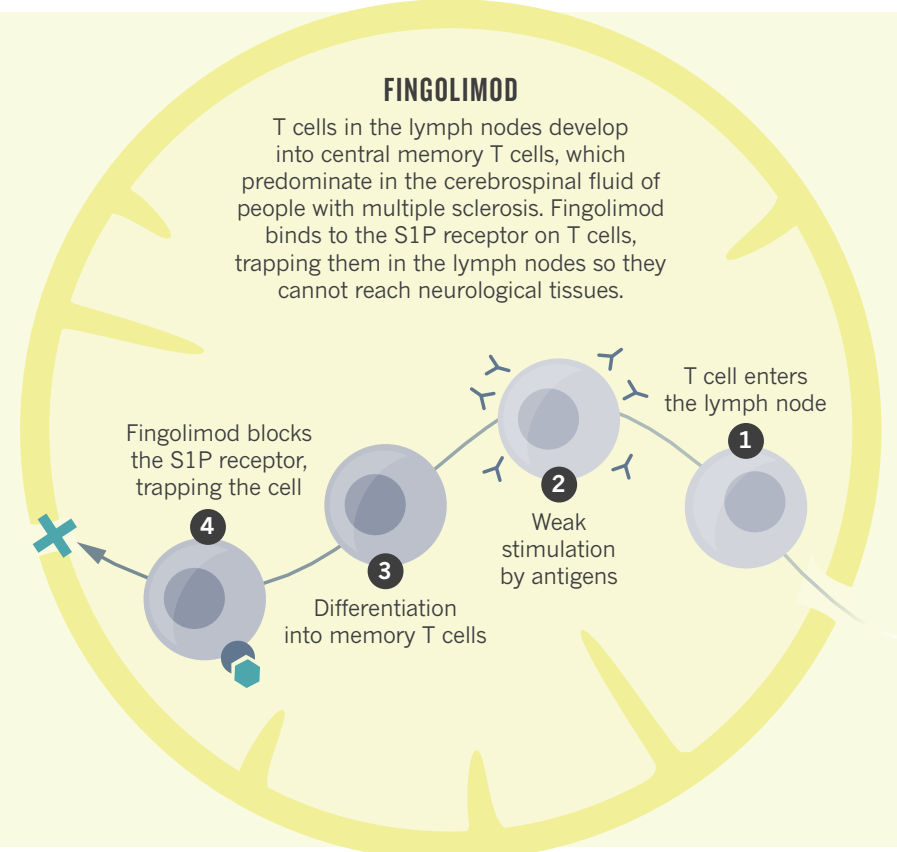

